\title{
Die Macht des Lokalen
}

\section{Social Entrepreneurship, das Konzept der Verwirklichungschancen und der soziale Mehrwert in der Sozialplanung}

\author{
Veronika Hammer
}

\begin{abstract}
Zur Fachlichkeit Sozialer Arbeit muss heute die Zusammenarbeit mit anderen Ressorts in einer Kommune gezählt werden. Angesichts nach wie vor bestehender Sparzwänge und gleichzeitig nicht weniger hohen Ansprüchen an die Maßnahmen- und Produktqualität ist stärker als früher Vernetzung und Schnittstellenmanagement angesagt.
\end{abstract}

Sozialplanung bietet Steuerungs-, Planungs- und Handlungskompetenzen im Kontext sozialer Infrastrukturplanung und aktiver Gesellschaftspolitik insbesondere auf kommunaler Ebene. Für die Profession der Sozialen Arbeit ist Sozialplanung von besonderer Bedeutung, weil sie sich auf die Bedürfnisse aller Bürger und Bürgerinnen im städtischen, gemeindlichen und landkreisweiten Bereich richtet. Sie will im optimalen Falle die Lebensqualität in ihren Planungsräumen verbessern, sodass gerade auch wirtschaftlich und sozial schwache Menschen mithilfe kommunaler Anreize ihre Ressourcen und Potenziale besser ausbauen und einbringen können. Sozialplanung versteht sich in diesem Sinne als integraler Bestandteil von Landkreis-, Stadt- und Gemeindeentwicklungsplanung.

Verschiedene Ressortplanungen der Schulentwicklung, des Bauens oder der Wirtschaft werden sich künftig verstärkt mit dem Sozialen verzahnen, denn angesichts hoher Sparzwänge und gleichzeitig nicht weniger hohen Ansprüchen an die Maßnahmen- und Produktqualität ist viel stärker als früher Vernetzung und Schnittstellenmanagement angesagt. Mit

Prof. Dr. Veronika Hammer lehrt

Sozialarbeitswissenschaft, gesellschaftswissenschaftliche Grundlagen und Methoden der empirischen Sozialforschung an der Fachhochschule Coburg.

E-Mail hammerve@fh-coburg.de einem abgegrenzten Ressortdenken kommen Professionelle aller Fachressorts heute nicht mehr weit. Die Fachlichkeit der Sozialen Arbeit und insbesondere der Sozialplanung ist daher gefordert, ihre moderierenden Qualitäten und Funktionen stärker als bisher einzusetzen. Bereits im Zuge der Einführung und Umsetzung des »Neuen Steuerungsmodells « konnte man - bezogen auf formale »Dezentralisierungsgrade", auf controllingorientierte Managementinstrumente zur OutputSteuerung (Kühn/Feldmann 2005) sowie auf Performanzveränderungen - in einigen »Modernisierungsinseln « (Bogumil u.a. 2007) bereits Teilsiege erringen.

Prospektiv betrachtet kann Sozialplanung beispielsweise an Einfluss gewinnen, wenn es ihr gelingt, ein integrierendes interdisziplinär-kommunikatives Netz zu bilden, das von einer Steuerungsgruppe mit sozialplanerischem Knowhow geleitet wird. Mit im Boot sind beispielsweise die regionalen Fach- und Sozialraumplanungen, Vertreterinnen und Vertreter von Stadtrats- oder Kreistagsfraktionen, Verwaltungskräfte, Profis aus freien Trägern, Arbeitskreise, ausgewählte sozialräumlich orientierte Institute sowie Firmen, Bürgerinitiativen, Organisationen und Selbsthilfeinitiativen in den Planungs- und Sozialräumen der jeweiligen Region.

Sozialer Raum ist zwar mehr als Planungsraum, er geht über diesen hinaus: mit den Biografien der Menschen, mit ihren beruflichen und privaten Mobilitäten, mit ihren Arbeitsbeziehungen, Freundschaften und Partnerschaften, die sich international oder national inszenieren. Dennoch ist es der Planungsraum, auf den sich die nahräumliche Planung beziehen wird.

In dieser Eingeschränktheit liegt jedoch auch die Stärke sozialplanerischer Intervention: Die Macht des Lokalen (Berking 2006) in einer Zeit multilokaler Netze verleiht eine Kraft der Identität, die nicht zu unterschätzen ist. Wenn das Lokale nicht als Muss-Gemeinschaft, sondern als Kann-Gemeinschaft und als Teil-Gesellschaft mit der Option bestmöglicher Differenzierungs- und Mobilitätschance verstanden wird, können dort mittels sensibler kommunaler Suchprozesse neue Ressourcen entdeckt und gebildet werden.

Dazu gehört es, neue Wege einzuschlagen, wenn es um die Lebensqualität der Menschen vor Ort geht. Stets ist es wichtig, auf soziale Ungleichheiten hinzuweisen, wo sie erkennbar oder verdeckt wahrnehmbar sind. Gleichzeitig sollte es jedoch gelingen, die Möglichkeiten in Betracht zu ziehen, die eine Kommune hat, um nahräumlich zu unterstützen, Partizipationswege anzubieten und Aktivierungsgelegenheiten zu schaffen. Integrierte Leitziele wie beispielsweise Gesundheit, Bildung oder Beschäftigung (Mardorf 2006) machen es leichter, mit innovativem Handling soziale Bezüge in Sozialberichterstattungen $\mathrm{zu}$ moderieren. Die Ressourcen und Fähigkeiten (»Capabilities «) der Bevölkerung können selbstverständlicher artikuliert und gezeigt werden als in einer Zone vorgegebener Bilder und statischer Bezüge.

\section{Konzept der Verwirklichungschancen}

Das Konzept der Verwirklichungschancen des Nobelpreisträgers Amartya Sen weist bereits heute international und verstärkt nun auch in Deutschland eine geeignete Referenz zu den aufgeworfenen Zusammenhängen auf. In der Konzeption von Sen (1999) sind die verschiedenen Freiheiten von Individuen die Grundbausteine.

»Daher gilt die Aufmerksamkeit vor allem der Erweiterung der ,Verwirklichungschancen $<$ der Menschen, genau das Leben führen zu können, das sie schätzen, und zwar mit guten Gründen. Die Verwirklichungschancen lassen sich durch 
öffentliche Maßnahmen vergrößern, doch lässt sich die Richtung solcher Maßnahmen durch wirksamen Einsatz partizipatorischer Verwirklichungschancen seitens der Öffentlichkeit ihrerseits beeinflussen. «(Sen 1999, S. 29).

Diese zweigleisige Beziehung ist für sozialplanerisches Denken und Handeln zentral bedeutend:

- weil substanzielle individuelle Freiheiten ein kritisches Potenzial haben, die den Erfolg einer Gesellschaft ausmachen (gesellschaftliche Wirksamkeit: Ergebnis- bzw. Outputorientierung)

- weil mehr Freiheit die Fähigkeit der Menschen stärkt, sich selbst zu helfen (soziale Wirksamkeit: Tätigkeit bzw. »agency « des Menschen)

Eine vermeintlich passive Bevölkerung, die nur durch den Einsatz der »richtigen « politischen Steuerungsmaßnahmen und Güter bedacht werden muss, wird auf einmal zu einer am Markt aktiv teilnehmenden Spezies, zu einem Teil der sozial, wirtschaftlich und politisch handelnden Öffentlichkeit.

Für Sozialplanung sind nach wie vor diejenigen Menschen und Gruppen von sensibler und erstrangiger Bedeutung, die unterversorgt sind, beispielsweise im Hin- blick auf Einkommen, Bildung, soziale Kontakte usw. Dazu zählen bekannterweise insbesondere Langzeitarbeitslose, Personen ohne Schulabschluss, chronisch Kranke usw. In diesen Fällen sind die individuellen, sozialen und wirtschaftlichen Chancen sowie die existenzielle Selbstän- tya Sen die Verwirklichungschancen die Möglichkeiten oder Fähigkeiten von Menschen, ein Leben führen zu können, für das sie sich mit guten Gründen entscheiden konnten und das die Grundlagen der Selbstachtung nicht in Frage stellt (vgl. Abb. 1).

\section{"Das Sozialwesen ist einer der größten Dienstleistungsbereiche der Republik«}

digkeit möglicherweise stark eingeschränkt. Aber ein rein auf die relative finanzielle Stellung bezogener Begriff von Unterversorgung reicht schon lange nicht mehr aus, um gleichzeitig auch als Grundlage für sozialplanerisches und sozialpolitisches Handeln dienen zu können. Denn teilweise verzichten Menschen bewusst auf gewisse Güter oder entscheiden sich bewusst gegen eine Teilnahme am Leben einer »Normalität". Daher sind im theoretischen Konzept von Amar-
Ein erster Bezug zum sozialplanerischen Umgang assoziiert bekannte Bezüge zum Lebenslagen-Ansatz, zu seinen Spielräumen und ressourcenorientierten Anreizen. Der besondere Charme des Capability-Konzeptes liegt in seinem internationalen Bekanntheitsgrad und in der dadurch entstehenden Chance, eine internationale Vergleichbarkeit wesentlicher Verwirklichungsaspekte zu erreichen.

Der professionelle Blick der Sozialplanung richtet sich einerseits auf die »indi-

\section{Verwirklichungschancen}

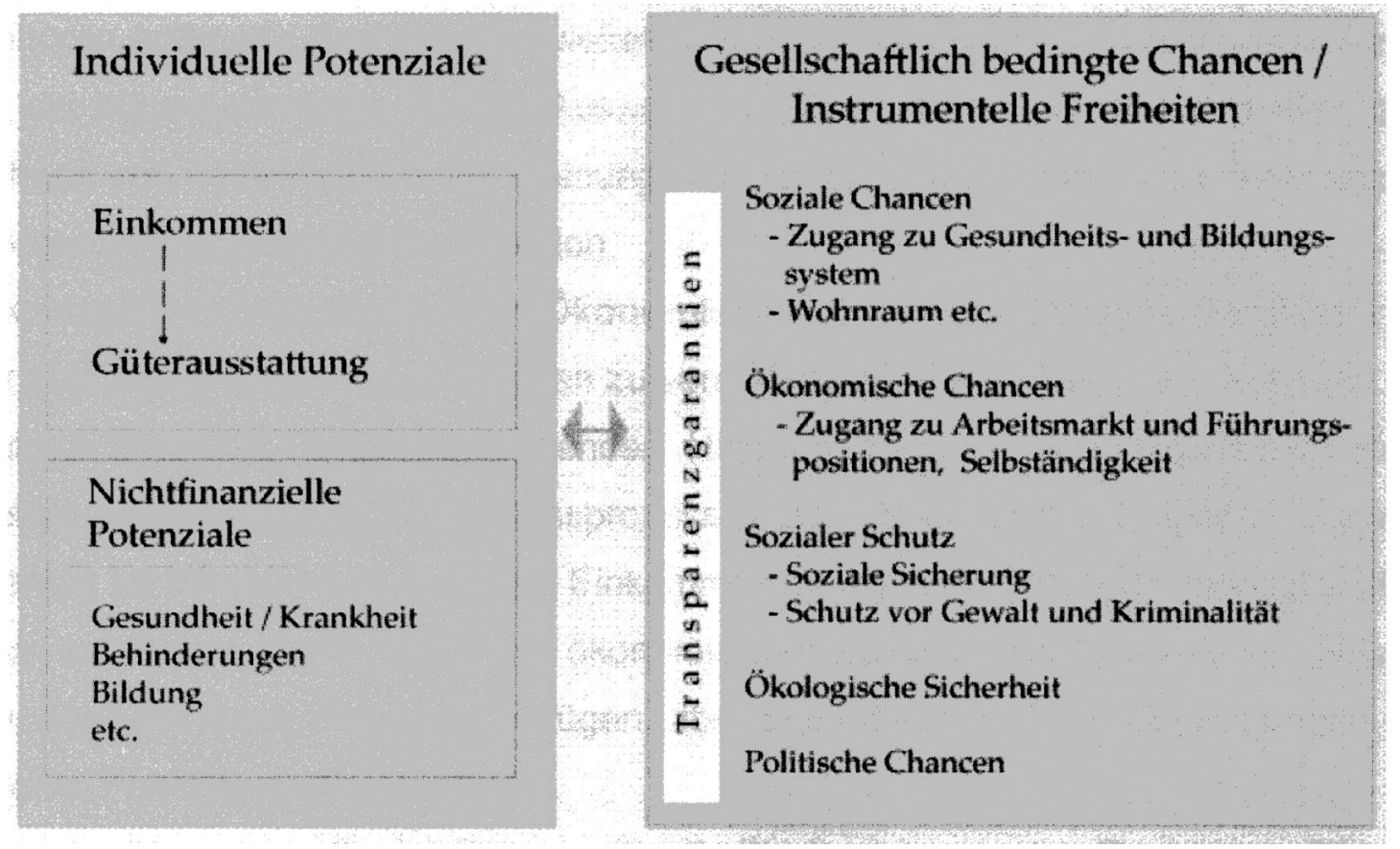

Abb. 1: Gelungene empirische Operationalisierungen des Konzeptes der Verwirklichungschancen von Amartya Sen beruhen auf zentralen Dimensionen der Verwirklichungschancen (Arndt u.a. 2006, S. 8, Abb. 1) 


\begin{tabular}{ll}
\hline Transparenzgarantien & $\begin{array}{l}\text { Gesellschaftlich bedingte Chancen/ } \\
\text { Instrumentelle Freiheiten (Beispiele) }\end{array}$ \\
\hline Soziale Chancen & $\begin{array}{l}\text { Schulen, Bildungseinrichtungen, Kinos, Cafés, } \\
\text { Netzwerke, Projekte, Initiativen, Klubs, } \\
\text { Vereine, Verbände, Sport, Freizeit }\end{array}$ \\
\hline Ökonomische Chancen & Beschäftigung in der Region, Unternehmen, \\
& Einzelhandel, Möglichkeiten zur Existenz- \\
& gründung, arbeitsmarktpolitische Programme \\
& (z. B. 50+) \\
\hline Sozialer Schutz & $\begin{array}{l}\text { Äter, Behörden, Zugang zu Transferleistun- } \\
\text { gen, niedrigschwellige soziale Dienste, Pflege- } \\
\text { dienste, Dienstleistungsservice }\end{array}$ \\
& Schutz durch Straßenbeleuchtung, durch \\
& Polizeikontrollen, durch Sorgfalt in der öffent- \\
& lichen Wahrnehmung von Bedrohungen \\
\hline Ökffentliches Nahverkehrsnetz, Abgaskontrol- \\
len, Initiativen zum Naturschutz, Kinderparks
\end{tabular}

Abb. 2: Transparenzgarantien und ihre Auswirkungen im Alltag

\section{Literatur}

Amt für Soziale Arbeit Wiesbaden (Burgmeier, K./Unkhoff, K./Lühning, E./Oberkirch, G.) 2006: Handbuch für stadtteilbezogene Soziale Arbeit. Wiesbaden: Materialien zur Sozialplanung. Arndt, C./Dann, S./Kleimann, R./Strotmann, H./Volkert, J. 2006: Das Konzept der Verwirklichungschancen (A. Sen). Empirische Operationalisierung im Rahmen der Armuts- und Reichtumsmessung. Machbarkeitsstudie. Hg.: BMAS. Reihe: Lebenslagen in Deutschland. (Internet http://www.bmas.bund. de)

Berking, Helmut (Hg.) 2006: Die Macht des Lokalen in einer Welt ohne Grenzen. Frankfurt am Main, New York: Campus Verlag.

Bogumil, Jörg/Grohs, Stephan/Kuhlmann, Sabine/Ohm, Anna, K. 2007: Zehn Jahre Neues Steuerungsmodell. Eine Bilanz kommunaler Verwaltungsmodernisierung. Sonderband 29. Modernisierung des öffentlichen Sektors. Berlin: edition sigma.

Gergs, Hans-Joachim 2007: Vom Sozialmanagement zum Social Entrepreneurship. Sozialen Mehrwert schaffen durch unternehmerisches Denken und Handeln. In: König, Joachim/Oerthel,
Christian/Puch, Hans-Joachim (Hg.): Mehrwert des Sozialen. Gewinn für die Gesellschaft. ConSozial 2006. München alliteraverlag. S. 173-185.

Kühn, Dietrich/Feldmann, Ursula (Hg.) 2005: Steuerungsunterstützung durch Sozialplanung und Controlling auf kommunaler Ebene. Hand- und Arbeitsbücher des Deutschen Vereins für öffentlich und private Fürsorge. Berlin: Eigenverlag des Deutschen Vereins.

Maier, Hugo/Zimmermann, Andreas 2005: Corporate Citizenship und Soziale Arbeit. Berufschancen für Wirtschaftssozialarbeiterinnen und -sozialarbeiter. In: Soziale Arbeit 7/2005. S.252-258.

Mardorf, Silke 2006: Methoden und Konzepte von Sozialberichterstattung eine empirische Analyse kommunaler Armuts- und Sozialberichte. Wiesbaden: VS-Verlag.

Schedler, Kuno/Proeller, Isabella 2006: New Public Management. Bern, Stuttgart, Wien: Haupt Verlag UTB.

Sen, Amartya 1999: Ökonomie für den Menschen. Wege zu Gerechtigkeit und Solidarität in der Marktwirtschaft. München, Wien: Carl-Hanser-Verlag. viduellen Potenziale « und andererseits auf die "gesellschaftlich bedingten Chancen «, auf die »instrumentellen Freiheiten «. Je nach Potenzialen (Einkommen, Güterausstattung, Gesundheit, Krankheit, Behinderung, Bildung etc.) bzw. je nach »Transparenzgarantien « (soziale Chancen, ökonomische Chancen, sozialer Schutz, ökologische Sicherheit, politische Chancen) kann kommunale Sozial(raum)planung ihr Set von Indikatoren bezogen auf Zielgruppen oder Quartiere usw. ausloten.

\section{Sozialer Mehrwert durch sozialplanerische Steuerung}

Der strukturelle Umbruch im Sozialwesen ist derzeit nicht abgeschlossen. Viele Ausgründungen in gGmbHs, Budgetierungen, Ambulantisierungen usw. fordern zu innovativem und kreativem Sozialplanen heraus. Sozialplanung muss Hindernisse überwinden und sich gegen Widerstände positiv durchsetzen. Dies kann dann gelingen, wenn unternehmerisches Denken und Handeln (»social entrepreneurship«, siehe auch Gergs 2007) in der sozialplanerischen Steuerung zunehmend eine Rolle spielen. Zusätzlich zum Social Management als administrativem Verhalten kommt nun unternehmerisches Verhalten als wettbewerbsorientiertes Verhalten, das zukunftsorientiert sozialen Mehrwert schaffen will.

Das »Sozialwesen « ist einer der größten Dienstleistungsbereiche in der Bundesrepublik, ein großer Wirtschaftszweig, beispielsweise in Behindertenhilfe, Jugendhilfe oder Pflege. Soziale Dienstleistungen werden im Zuge des demografischen Wandels, der Kürzungen im sozialstaatlichen Transferleistungssystem usw. in Zukunft noch stärker nachgefragt werden. Die Sozialverantwortung von öffentlichen und freien Trägern Sozialer Arbeit sowie von Unternehmen wird steigen müssen.

Sozialplanung erhält zunehmend die Aufgabe, die neuen Unternehmens- und Bürgerbeteiligungsaktivitäten (»Schnittstellenmanagement ", siehe auch Maier/Zimmermann 2005) mit aufzugreifen, sie zu erspüren und Richtungsentscheidungen zu treffen. Unternehmen beteiligten sich beispielsweise zunehmend an »Corporate Citizenship Aktivitäten«, d.h. sie entwickeln Konzepte zur ethisch fun- 
dierten unternehmerischen Gemeinwohlorientierung. Kommunale und regionale Belange werden als Standortvorteil gesehen. Von daher werden Partner auf allen Seiten gesucht. Sozialplanung könnte beispielsweise mit der Entdeckung und Entwicklung von Kooperationsprojekten, die den Einbezug des Privaten in die öffentliche Aufgabenerbringung transportieren, mit dabei sein und erfolgreiche Public-Private-Partnership-Projekte (Schedler/Proeller 2006, S. 204 ff.) ins Leben rufen.
Die größere Vielfalt der Angebote in den Regionen forciert sowohl Wettbewerb als auch Konkurrenz im sozialen Dienstleistungsbereich. In diesem Umbruchprozess werden neue Unternehmensformen und sozialplanerische Steuerungsmöglichkeiten im Sozialwesen entstehen. Wenn es gelingt, soziale Innovationen in die bestehenden und in die sich neu entfaltenden sozialen Organisationen $\mathrm{zu}$ integrieren, dann ist unternehmerisches sozialplanerisches Handeln angekommen.

\section{Transparenzgarantien von} Kommunen - partizipatorische Verwirklichungschancen der Öffentlichkeit

Auf der Seite der Kommunen prüft Sozialplanung konsequenterweise $\mathrm{ab}$, welche öffentlichen Maßnahmen bereits ergriffen wurden, um soziale, ökonomische und politische Chancen sowie sozialen Schutz und ökologische Sicherheit möglichst als »Transparenzgaran-

\section{Monitoring}

In der Rubrik »Monitoring « nehmen Autorinnen und Autoren einzelne Arbeits- und Themenfelder der Sozialen Arbeit in den Blick um zu untersuchen, was sich dort Neues tut oder warum dort Praxis und Theorie auf der Stelle treten. Die Artikel sind eine aktuelle Ergänzung zu den wechselnden Schwerpunktthemen aus der Sozialen Arbeit in den Blättern der Wohlfahrtspflege. Eine Übersicht der bisherigen Beiträge in der Rubrik »Monitoring «:

Sorgenkind lokale Ökonomie

Ergebnisse der dritte Befragung in den Programmgebieten der

»Sozialen Stadt «

Von Christa Böhme und Thomas Franke

Blätter der Woblfahrtspflege 5/2007

Orientierungslos, mutlos, machtlos

Die Idee der Bürgergesellschaft hat es noch nicht zum gesellschaftlichen Leitbild geschafft - Das liegt auch an uns selbst

Von Stefan Nährlich

Blätter der Woblfahrtspflege 4/2007

Synergielösungen für Sozialräume

Plädoyer für Fusionen kleiner Träger

Von Armin Wöhrle

Blätter der Wohlfahrtspflege 4/2007

Das Betreuungsgesetz droht zu scheitern

Erforderlich sind eine konsequenteProfessionalisierung, mehr Kompetenzen für die Betreuungsbehörden, obligatorische Sozialgutachten sowie eine regelmäßige Berichterstattung

Von Wolf Crefeld

Blätter der Wohlfahrtspflege 3/2007

Wider die Individualisierung der Selbsthilfe

Karriere eines strapazierten Begriffs - Beispiel: Selbsthilfe

Wohnungsloser

Von Stefan Gillich

Blätter der Woblfahrtspflege 3/2007

Auf der Suche nach der guten Praxis

Die Qualitätsentwicklung in der Sozialen Arbeit — ein Überblick

Von Andrea Buckley und Karl-Heinz Boeßenecker

Blätter der Wohlfahrtspflege 2/2007

Distanzierte Nähe

Wie Wohlfahrtsverbände und traditionelle Milieus in Ostdeutschland (nicht) in Beziehung stehen

Von Peter-Georg Albrecht

Blätter der Wohlfahrtspflege 2/2007
Was Fachkräfte wissen müssen

Der Qualifikationsrahmen Soziale Arbeit

Von Peter Buttner und Ulrich Bartosch

Blätter der Wohlfahrtspflege 1/2007, Seite 28-30

Das Format mit Inhalt füllen

Interview mit Wolf Rainer Wendt zum »Qualifikationsrahmen Soziale Arbeit«

Blätter der Woblfahrtspflege 1/2007, Seite 29

Eltern stützen, Kinder schützen

Nach den Gesetzesänderungen:

Wie zukunftsfest ist das Kinder- und Jugendhilferecht? Von Johannes Münder

Blätter der Wohlfahrtspflege 1/2007, Seite 31-32

Alles wird zur Dienstleistung

Die wirkungsorientierte Finanzierung von Jugendhilfe

Von Benjamin Landes

Blätter der Woblfahrtspflege 1/2007, Seite 33-34

Schulsozialarbeit braucht mehr Rechte

Ein Berufsfeld der Sozialen Arbeit zwischen Lerntradition und Lebenswelt

Von Susanne Hartmann-Hanff

Blätter der Woblfahrtspflege 1/2007, Seite 35-36

Qualitätssicherung zivilisiert den Wettbewerb

System und Arbeitsfelder im Vergleich

Von Roland Schmidt

Blätter der Wohlfahrtspflege 6/2006, Seite 230-232

Indikatoren gesucht

Neue Ansätze zur Integrationssteuerung in Deutschland

Von Kristin Schwarze

Blätter der Wohlfahrtspflege 6/2006, Seite 233-234

Die armen Kinder

Junge Menschen als die unsichtbaren Verlierer von Hartz IV

Von Gerda Holz

Blätter der Wohlfahrtspflege 5/2006, Seite 193-195

Der Jahresbezugspreis beträgt 58,- Euro (für Studierende und arbeitslose Bezieher auf jährlichen Nachweis 29,-Euro).

Die Einzelhefte können zum Preis von 11,- Euro zuzüglich Versandkosten und Mehrwertsteuer bezogen werden bei:

Nomos Verlagsgesellschaft, 76520 Baden-Baden, Telefon 07221 2104-39, Fax 07221210443,

E-Mail hohmann@nomos.de 
tie« zu gewährleisten. Ebenfalls wird geklärt, welche wirksamen partizipatorischen Verwirklichungschancen die Menschen vor Ort eigentlich haben. Können sie beispielsweise diese Maßnahmen bewerten, sie prüfen und in Einklang mit ihren Interessen und Fähigkeiten bringen? Womöglich bringen sie bereits ihre Kompetenzen und Potenziale in ein Netzwerk von sozialen und kulturellen Möglichkeiten vor Ort ein und sind in der Lage, dies noch zu steigern oder zu differenzieren. Neue Ideen seitens der Bürgerinnen und Bürger und seitens der Stadt-, Landkreis- oder Gemeindeverantwortlichen in der Sozialplanung sind gefragt (vgl. Abb. 2).

Je nach individuellen materiellen Potenzialen (Einkommen, Güterausstattung) und nichtfinanziellen Potenzialen (Gesundheit, Krankheit, Behinderung, Bildung usw.) können die Bürgerinnen und Bürger dann in Korrespondenz mit den zur Verfügung gestellten gesellschaftlich bedingten Chancen und instrumentellen Freiheiten agieren. Sozialplanung hätte dann die Aufgabe darauf hinzuweisen, wo noch auf Seiten der Kommunen und der Individuen ein Mangel an Verwirklichungschancen existiert und wie darauf hingewirkt werden kann, dass hier weitere oder neue öffentliche und individuelle Maßnahmen ergriffen werden.

Methodisch betrachtet kann Sozialplanung durchaus auf einen gut ausgestatteten Methodenpool der stadtteilbezogenen Sozialen Arbeit zugreifen (z. B. Amt für Soziale Arbeit Wiesbaden 2006), der durch die professionellen Entrepreneure in der Sozialplanung zur Weiterentwicklung und Schaffung sozialen Mehrwertes neu kontextuiert werden kann.

\section{Soziale Arbeit und Wohnungswirtschaft}

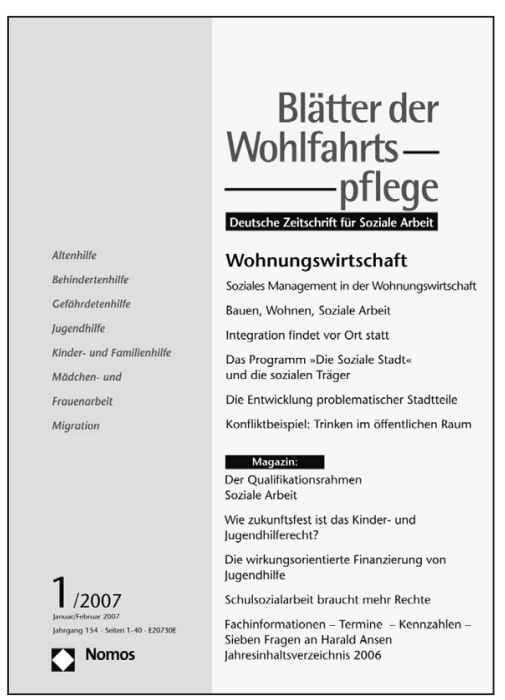

Soziale Arbeit in der Wohnungswirtschaft gehört zu den Tätigkeitsfeldern, die in den letzten Jahren einen beeindruckenden Aufschwung erfahren haben. Vielfach unbemerkt von der Öffentlichkeit - und auch von der Profession selbst - haben sich in Wohnungsunternehmen Konzepte der Mieterbetreuung und der Stadtteilentwicklung durchgesetzt, die ihre Herkunft aus der Sozialen Arbeit und der Gemeinwesenarbeit nicht verleugnen können. Das Themenheft »Wohnungswirtschaft « der Blätter der Wohlfahrtspflege 1/2007 beleuchtet diese Entwicklung in verschiedenen Beiträgen.

Der demografische Wandel, die strukturelle Arbeitslosigkeit, die Folgen der Migration, die sozioökonomische Polarisierung angesichts gesetzlicher und wirtschaftlicher Entwicklungen - alle diese gesellschaftlichen Trends wirken unmittelbar auf den Wohnungsmarkt ein. Eine Untersuchung in Bayern hat bereits vor einigen Jahren gezeigt, dass sich zahlreiche Wohnungsunternehmen bereits mit dem sozialen Management ihrer Immobilien beschäftigen, dass aber auch das Problemniveau zunimmt. Viele Wohnungsunternehmen leisten danach bereits erhebliche Beiträge, um die sozialen Folgen von gesellschaftlichen Veränderungsprozessen zu bewältigen, und entlasten dadurch auch die kommunale und staatliche Sozialpolitik.

Eine Kooperation von Wohnungswirtschaft und Sozialer Arbeit könne gerade in den Zeiten neuer Armut und sozialer Segregation eine erfolgversprechende Zukunftsstrategie, schreiben Clemens Klikar und Dieter Ruhnke in ihrem Erfahrungsbericht aus Berlin in den Blättern der Wohlfahrtspflege 1/2007. Die Mieter und Bürger werden durch solche Kooperationen mit ihrer Kompetenz eingebunden in wesentliche Entscheidungsprozesse bei der Gestaltung ihrer persönlichen Wohnsituation.

Das Einzelheft der Blätter der Wohlfahrtspflege 1/2007 ist zum Preis von 13,- Euro bei der Nomos Verlagsgesellschaft in Baden-Baden erhältlich (E-Mail hohmann@ nomos.de).

\section{Gemeinsame Servicestellen}

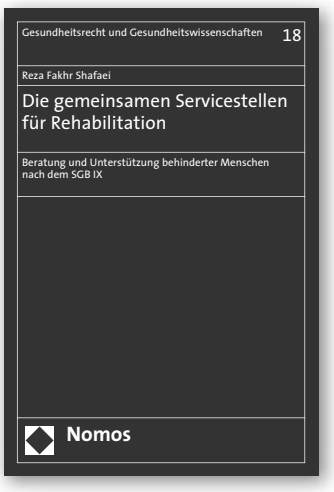

\section{Die gemeinsamen Service- stellen für Rehabilitation} Beratung und Unterstützung behinderter Menschen nach dem SGB IX

Von RA Dr. Reza Fakhr Shafaei 2008, 276 S., brosch., ca. 54, $-€$ ISBN 978-3-8329-3207-7

(Gesundheitsrecht und Gesundheitswissenschaften, $B d$. 18)

Erscheint Januar 2008

Mit dem SGB IX Rehabilitation und Teilhabe behinderter Menschen sind am 1. Juli 2001 auch die Regelungen der \$§22-25 SGB IX über die Gemeinsamen Servicestellen der Rehabilitationsträger in Kraft getreten. Der Autor reagiert mit einem umfassenden Ansatz auf diese neue Entwicklung im deutschen Behindertenrecht. Die zahlreichen und bisher ungeklärten Rechtsfragen werden einer praxisgerechten Lösung zugeführt.

Das Werk dient zugleich als „,Handbuch" für alle, die auf dem Gebiet des Behinderten- und Rehabilitationsrechts wissenschaftlich und praktisch tätig sind.

\section{Nomos}

Bitte bestellen Sie bei Ihrer Buchhandlung oder bei Nomos | Telefon 07221/2104-37 | Fax -43 | www.nomos.de | sabine.horn@nomos.de 